\title{
Aprendizado de Máquina nos Jogos para Medicina: Uma Revisão Sistemática
}

\author{
Jesaías Carvalho Pereira Silva ${ }^{1}$, Everton Jales de Oliveira ${ }^{1}$, Francisco Milton Mendes Neto ${ }^{1}$, \\ Araken de M Santos ${ }^{1}$, Paulo Gabriel G Queiroz ${ }^{1}$, Alexandre Adler Cunha de Freitas ${ }^{1}$ \\ ${ }^{1}$ Programa de Pós-Graduação em Ciência da Computação - Centro de Ciências Exatas e Naturais \\ Universidade Federal Rural do Semi-Árido (UFERSA) \\ BR 110 - KM 47 - 59625-900 - Mossoró/RN - Brasil \\ \{jesayassilva,verto.jales\}@gmail.com, \\ \{miltonmendes, araken, pgabriel\}@ufersa.edu.br, alexandreadler@outlook.com
}

\begin{abstract}
This work presents a Systematic Literature Review of Machine Learning Literature in Games for Medicine held in 4 international research sources in the last 10 years, whose objective is to identify the state of the art of machine learning in medical games. As a result of the Systematic Review presented in this study, 1040 papers were analyzed, of which 40 were pre-selected and 12 were selected for data extraction. Among the selected works, it was possible to list several techniques of machine learning, types of learning, health areas and games used today.
\end{abstract}

Resumo. Este trabalho apresenta a condução de uma Revisão Sistemática da Literatura sobre o Aprendizado de Máquina em Jogos para medicina realizada em 4 fontes de pesquisa internacionais nos últimos 10 anos, cujo objetivo é identificar o estado da arte do aprendizado de máquina nos jogos para medicina. Como resultado da Revisão Sistemática apresentada neste trabalho, foram analisados 1040 trabalhos, e desse total, 40 foram pré-selecionados e 12 foram selecionados para extração de dados. Entre os trabalhos selecionados, foi possivel elencar várias técnicas de aprendizado de máquina, tipos de aprendizado, áreas da saúde e jogos utilizados na atualidade.

\section{Introdução}

O uso do Aprendizado de Máquina (AM) é importante para aplicações práticas de Inteligência Artificial por conta da indução (Luger, 2014). A indução é a forma de inferência lógica que permite que conclusões gerais sejam obtidas de exemplos ou observações particulares. Assim, o AM é programado para aprender com a experiência passada usando o princípio da inferência chamado de indução, a partir do qual se obtém conclusões genéricas a partir de um conjunto de exemplos (Faceli, Lorena, Gama, \& Carvalho, 2011).

Na medicina, o AM pode ser aplicado a um conjunto de dados com o propósito de desenvolver modelos de riscos robustos (Deo, 2015), o que não é surpreendente, pois podem ser aplicados a uma ampla gama de campos (Deo et al., 2014), facilmente reduzindo a tarefa de prever resultados de diversos recursos ou encontrando padrões recorrentes em conjunto de dados multidimensionais. A aplicação do AM na medicina enfrenta alguns obstáculos como da precisão realizada por um especialista humano em relação á máquina (Deo, 2015). 
No desenvolvimento dos jogos sérios, a inteligência artificial, por meio do AM, oferece um potencial significativo, aprimorando a experiência do jogador em todas as etapas do jogo, ajudando a melhorar vários estágios dentro do desenvolvimento de jogos sérios (Frutos-Pascual \& Zapirain, 2017). Os jogos sérios apresentam características explícitas e cuidadosamente pensadas com propósitos educacionais e não se destinam a serem jogados somente para diversão (Abt, 1987). Segundo Clua (2014), o uso de jogos sérios para fins relacionados à saúde tem se tornado uma forte tendência e o seu impacto pode abrir formas de tratamento sem precedentes.

O AM pode ser usado de diversas maneiras na medicina como previsão de crises e doenças. Os jogos para medicina são de grande valia e também podem auxiliar de diversas maneiras, tais como: treinamento de médicos; identificação de doenças; e, tratamento de doenças. Para desenvolver um jogo para medicina é possível utilizar o AM. Entretanto existem várias tipos, técnicas e tarefas de AM existentes. Assim, surgiu a necessidade de se descobrir quais as principais formas de se aplicar o AM para desenvolver jogos para medicina.

Diante disso, nesta Revisão Sistemática Literatura (RSL), são apresentados os jogos, técnicas, tarefas e áreas médicas explorados pelo AM. O restante deste trabalho está organizado da seguinte forma: na Seção 2, apresenta-se o Processo Sistemático; na Seção 3 , apresenta-se a Condução da Revisão Sistemática; na Seção 4, apresentase os Resultados da Revisão Sistemática e Discussões; e, na Seção 5, apresenta-se as Conclusões e Limitações desta pesquisa.

\section{Processo Sistemático}

As RSL são consideradas estudos secundários, que têm sua fonte de dados nos estudos primários (Galvão \& Pereira, 2014). Por meio da RSL é possível identificar, avaliar e interpretar toda a pesquisa disponível relevante para uma questão especifica ou área temática, ou fenômeno de interesse de forma repetitiva e imparcial (Kitchenham, 2004). Nesta seção, são apresentados os detalhes da metodologia utilizada na RSL, que consiste em: definir os objetivo da revisão; definir as questões de pesquisa; definir a string de busca, definir as bases de dados; definir os critérios de inclusão, exclusão e qualidade; definir os procedimentos de seleção dos estudos; e, definir a extração dos dados.

\subsection{Objetivos e Questões de Pesquisa}

O objetivo desta RSL é identificar o estado da arte da utilização do AM nos jogos para medicina, por meio da identificação e análise de jogos que utilizam AM com aplicações na medicina. A questão primária de pesquisa a ser tratada neste trabalho é "Como o aprendizado de máquina está sendo utilizado nos jogos para medicina?". Essa questão foi decomposta em outras questões de pesquisa, que buscam alcançar o objetivo proposto.

- Questão secundária (QS1): Qual o principal tipo de aprendizagem de máquina utilizado nos jogos para medicina?

- Questão secundária (QS2): Quais as principais técnicas de aprendizagem de máquina nos jogos para medicina?

- Questão secundária (QS3): Quais os principais jogos sérios que utilizam aprendizagem de máquina na área da medicina?

- Questão secundária (QS4): Quais as principais áreas da medicina em que os jogos encontrados estão sendo aplicados? 


\subsection{Definição da String de Busca}

Para definir a string de busca, é importante encontrar as palavras chave relacionadas a pesquisa. As palavras chave nas Revisões Sistemáticas são definidas por meio das questões de pesquisa sob análise. Inicialmente, foram definidas as palavras chave que seriam utilizadas na string de busca, e posteriormente realizou-se uma busca piloto. Concluiu-se que as palavras deveriam ser todas no idioma inglês e que alguns sinônimos fossem adicionados. Na Tabela 1, são apresentadas as palavras chaves e sinônimos da estratégia de busca em Inglês.

Tabela 1. Palavras Chaves e Sinônimos

\begin{tabular}{|l|c|}
\hline Palavra Chave & Sinônimos \\
\hline Machine learning & Automatic learning, Automatically learning, Artificial Inteligence \\
\hline Medicine & Medic, Health \\
\hline Game & Games, Simulator, Simulation \\
\hline
\end{tabular}

Após realizar as combinações das palavras chaves e sinônimos foi definida a seguinte string de busca:

(“Machine learning” OR “Learning automatically” OR “Automatic learning” $O R$ “Artificial Inteligence”) AND (Games OR Game OR Simulator OR Simulation) AND (Medicine OR Medical OR Health)

\subsection{Bases de Dados}

Nesta pesquisa foram utilizadas bases de dados eletrônicas indexadas e máquinas de busca eletrônica, conhecidas internacionalmente que indexam trabalhos da grande área da computação ou interdisciplinar. Com base nesses critérios, as bases de dados escolhidas são IEEE, ACM Digital Library, Science Direct e Scopus.

\subsection{Critérios de Inclusão, Exlusão e Qualidade}

Os critérios de inclusão e exclusão são definidos para garantir a imparcialidade na condução da RSL. Trabalhos aceitos para análise devem atender a, pelo menos, um critério de inclusão. Foram definidos 2 critérios para inclusão (CI) de trabalhos, conforme apresentados a seguir.

- CI1: Trabalhos que apresentam técnicas de aprendizagem de máquina para jogos eletrônicos na área da medicina.

- CI2: Trabalhos que apresentam jogos eletrônicos que utilizam aprendizagem de máquina na área da medicina.

Trabalhos excluídos da análise devem atender a, pelo menos, um critério de exclusão. Foram definidos 3 critérios para exclusão (CE) de trabalhos, conforme apresentados a seguir.

- CE1: Trabalhos que não apresentam técnicas de aprendizagem de máquina;

- CE2: Trabalhos que não apresentam jogos eletrônicos;

- CE3: Trabalhos que não são relacionados à medicina.

Trabalhos com qualidade devem atender a todos os critérios de qualidade. Foram definidos 3 critérios para seleção de trabalhos com qualidade (CQ), conforme apresentados a seguir. 
- CQ1: Trabalhos publicados em jornais, revistas ou conferências com qualis igual ou acima de B5;

- CQ2: Trabalhos que apresentem 4 páginas ou mais;

- CQ3: Trabalhos escritos no idioma Inglês ou Português.

\subsection{Procedimento para Seleção dos Estudos}

O procedimento para seleção de estudos foi definido em 3 fases conforme detalha-se a seguir:

- Fase 1: Corresponde à busca e coleta de trabalhos. Nesta fase, as strings de busca formadas pela combinação dos sinônimos das palavras-chave identificadas são submetidas às máquinas de busca selecionadas e os trabalhos encontrados foram coletados.

- Fase 2: Corresponde à leitura dos resumos. Nesta fase, entre os trabalhos retornados são lidos os resumo e títulos, e constatando-se a relevância de um trabalho, com base em algum dos critérios de inclusão, o trabalho é foi pré-selecionado para ser lido na íntegra. Os trabalhos que se encaixaram em algum dos critérios de exclusão são excluídos, e caso não se encaixaram em nenhum também são pré-selecionados.

- Fase 3: Corresponde à leitura do trabalho completo. Os trabalhos que foram aprovados na etapa anterior são lidos na integralmente, novamente aplicados os CI e CE, e também aplicado o CQ. Os trabalhos aceitos nesta fase são utilizados para responder as questões de pesquisa.

\subsection{Extração de dados}

A seguir, são apresentados os dados extraídos dos trabalhos selecionados. Os dados são: título; ano; base de dados; fonte qualis; idioma; páginas; objetivo do trabalho; nome do jogo; temática abordada da saúde; forma do jogo ser visualizado; tipo de AM; técnica do AM; tarefa de AM; forma que o AM foi usado; ferramenta que foi usada para o AM; eficiência; vantagens; impacto; limitações; e, como o trabalho foi validado.

\section{Condução da Revisão Sistemática}

Nesta seção, são apresentados os resultados obtidos com as buscas dos estudos primários. As buscas nas bases de dados foram realizadas entre agosto e novembro de 2018 e foram limitadas aos trabalhos publicados entre os anos de 2008 e 2018. As buscas nas bases de dados foram realizadas por meio de Advanced Search, sendo adaptadas as particularidades de cada base.

Tabela 2. Resultados das buscas

\begin{tabular}{|l|c|c|c|}
\hline Base & Fase 1 & Fase 2 & Fase 3 \\
\hline IEEE & 363 & 18 & 6 \\
\hline ACM Digital Library & 313 & 9 & 2 \\
\hline Science Direct & 25 & 1 & 0 \\
\hline Scopus & 340 & 12 & 4 \\
\hline Total & 1040 & 40 & 12 \\
\hline
\end{tabular}

Na Fase 1 foram encontrados 1040 trabalhos, entre os quais 146 eram trabalhos repetidos. Na fase 2 foram selecionados 40 trabalhos após leitura dos resumos. Na Fase 
3, após a leitura completa e baseado nos critérios de inclusão e qualidade, foram selecionados 12 trabalhos. A seguir, pode-se observar na Tabela 2 os detalhes em relação ao quantitativo dos trabalhos em cada fase.

Observa-se que a base de dados da IEEE registrou o maior número de trabalhos selecionados nesta pesquisa, com 6 trabalhos. Os trabalhos selecionados foram publicados entre os anos de 2011 e 2018, em 2013 foram encontrados 4 trabalhos, sendo o maior número de trabalhos encontrados por ano nessa revisão sistemática, seguido por 2018 com 3 trabalhos selecionados.

\section{Resultados e Discussões}

Os trabalhos que foram selecionados têm o potencial de auxiliar a responder aos questionamentos que nortearam essa pesquisa. A sua principal questão é: Como o AM está sendo utilizado nos jogos para medicina? E a partir dessa questão surgiram as questões secundárias que são apresentadas e respondidas nas subseções a seguir.

\subsection{Qual o principal tipo de aprendizagem de máquina utilizado nos jogos para medicina?}

Dentre os tipos de AM existentes, os mais conhecidos são o Aprendizado Supervisionado (AMS), Aprendizado Não Supervisionado, Aprendizado Semissupervisionado e o Aprendizado por Reforço.

No AMS é fornecido ao algoritmo de aprendizado, ou indutor, um conjunto de exemplos de treinamento para os quais o rótulo da classe associada é conhecido (Monard \& Baranauskas, 2003). Esse AM tem como objetivo encontrar um modelo ou hipótese, a partir de dados de treinamento que podem ser utilizados para prever um rótulo ou valor que caracterize um exemplo novo, com base nos atributos de entrada (Faceli et al., 2011). Já na Aprendizagem Não Supervisionada segundo Norvig e Russel (2014), o agente aprende padrões na entrada, embora não seja fornecido nenhum feedback explícito. A forma mais comum de se usar o aprendizagem não supervisionada é no agrupamento: a detecção de grupos de exemplos de entrada potencialmente úteis.

No Aprendizado Semissupervisionado são usados exemplos rotulados e não rotulados, pois no AMS às vezes é trabalhoso, lento e custoso conseguir dados rotulados e usar o aprendizado não supervisionado pode não ser trivial para descobrir o conceito embutido nos nós dos clusters encontrados. Como é difícil solicitar a um especialista que classifique todos esses dados, ainda é possível solicitar ao especialista que classifique alguns desses dados com alta certeza. Assim, é possível utilizar algoritmos de Aprendizado Semissupervisionado (Matsubara, 2004). O Aprendizado por Reforço é apresentado por Faceli et al. (2011) como um AM na qual a meta é reforçar ou recompensar uma ação considerada positiva e punir uma ação considerada negativa. Um exemplo de Aprendizado por Reforço é o de ensinar um robô a encontrar a melhor trajetória entre dois pontos. Algoritmos de aprendizado utilizados nessa situação, em geral, punem a passagem por trechos pouco promissores e recompensam a passagem por trechos promissores.

Os trabalhos encontrados por meio da string de busca foram divididos entres esses AM. Na Figura 1, é apresentada a distribuição do uso dos tipos de AM nos trabalhos aceitos. 


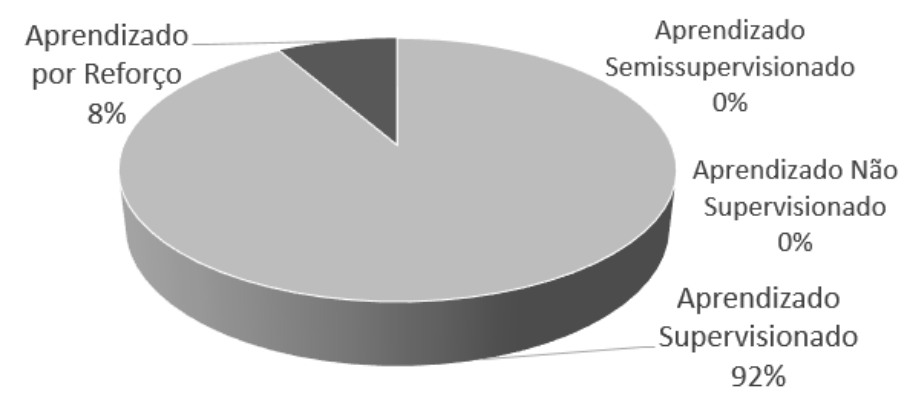

Figura 1. Tipo de Aprendizado de Máquina

O trabalho apresentado por Chouhan et al. (2015) utilizou o AMS, com o objetivo de aprender e prever os níveis de atenção dos jogadores para o próximo instante. Esse aprendizado se mostrou eficaz para realizar esta previsão. Entretanto, o trabalho possivelmente apresenta um erro no método de Entropia. No trabalho apresentado por Heller et al. (2013), foi utilizado o AMS para identificar o Transtorno do Déficit de Atenção com Hiperatividade (TDAH) dos jogadores. O aprendizado se mostrou eficaz com $70 \%$ de acerto, entretanto outros jogos apresentam maior nível de acerto como Brief Rating Scale descrito pelos autores.

Ahmad et al. (2013) utilizaram o AMS para tentar aprender e encontrar o caminho ideal em microcirurgias realizadas por especialistas no simulador. O aprendizado se mostrou relativamente médio em eficiência. Seu desempenho não foi muito encorajador para o exercício de agarrar em microcirurgias, provavelmente pelos poucos dados de treinamento, o que pode ser melhorado com a inclusão de mais dados de treinamento.

No trabalho apresentado por Alchalabi et al. (2017), o Aprendizado Supervisionado foi usado na representação dos modelos de níveis de atenção dos usuários. O aprendizado se mostrou eficiente na classificação dos dados de Eletroencefalografia (EEG) dos usuários, entretanto, foram usados poucos números de amostras e apenas de usuários saudáveis e não de pessoas diagnosticadas com TDAH, o que era objetivo do trabalho.

Alchalabi et al. (2018) apresentaram o mesmo aprendizado do trabalho mencionado anteriormente, mas foram apresentados novos resultados e validações, o que demonstrou uma taxa de $96 \%$ de acerto na classificação dos dados do EEG para detectar o estado de atenção correto durante o jogo em indivíduos saudáveis, e de $98 \%$ na classificação dos dados do EEG para detectar o estado de atenção correto durante o jogo em indivíduos com TDAH.

No trabalho apresentado por Hervés et al. (2016), os autores usaram o AMS para detecção da interação empática e não empática, possibilitando o diagnóstico de Distúrbios da Comunicação Social. O seu uso teve bom desempenho somente com dados sintéticos, e está longe de representar uma solução definitiva para fornecer suporte no diagnóstico de Distúrbios da Comunicação Social, o que pode ter acontecido pelo pequeno conjunto de treinamento.

O Aprendizado por Reforço foi utilizado em um trabalho apresentado por Perdiz et al. (2018), que foi utilizado a fim de intermediar possíveis ações do usuário e instruções de atuação do jogo, e tentar adaptar sua resposta para maximizar os resultados e assim, chegar ao melhor resultado no jogo. Apesar dos jogadores não apresentarem um efeito 
perceptível nos scores, o uso da técnica de aprendizado por reforço pode ser tomada ao projetar jogos controlados por biosignal com pessoas com deficiência e Assistive Living em mente.

Os trabalhos apresentados por Lima et al. (2016), Zielke et al. (2018), Flores et al. (2013), George et al. (2011) e Kim et al. (2013) não informaram o tipo de aprendizado utilizado. Entretanto, acredita-se que esses trabalhos usam o AMS, pois descrevem que o aprendizado pode aprender com experiências passadas, ou que foi utilizado dados de treinamento, o que se trata de uma característica do AMS.

Dessa forma, pode-se observar que o Aprendizado Supervisionado é o mais usado nos jogos para medicina, pois foi utilizado por $92 \%$ dos trabalhos. O AMS tem uma precisão que é necessária ser utilizada nesses jogos a fim do benefício de indivíduos e/ou jogadores e/ou pacientes, que se caracteriza por meio do treinamento dos dados no AM. Não foi identificado o uso de aprendizado não supervisionado e semissupervisionado nestes trabalhos. Apesar do aprendizado por reforço ter representado $8 \%$ do total de trabalho selecionados, ele pode se tornar uma alternativa viável nos jogos para medicina.

\subsection{Quais as principais técnicas de aprendizagem de máquina nos jogos para medicina?}

Seis dos trabalhos analisados especificaram as técnicas de AM usadas. Na Figura 2, é apresentada a porcentagem de uso das técnicas nos trabalhos selecionados.

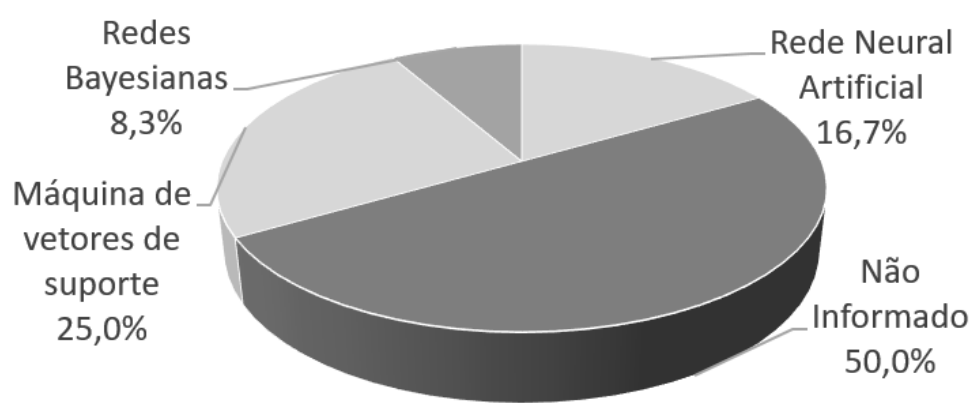

Figura 2. Técnicas de Aprendizado de Máquina

A Máquina de Vetores de Suporte (MVS) é uma técnica de AMS usado para classificação de dados binários linearmente não probabilísticos. As MVS têm a capacidade de ajustar um hiperplano que separa duas classes diferentes, funcionam bem com dados não linearmente separáveis usando o truque do kernel que mapeia as entradas de dados para espaços dimensionais mais altos de recursos nos quais eles são facilmente separáveis (Alchalabi et al., 2017). A MVS foi usada em 25\% dos trabalhos analisados. Por Alchalabi et al. (2017) (2018) ela foi usada com a tarefa de Regressão Linear, enquanto Hervás et al. (2016) utilizaram a MVS com Regressão logística, que é outra forma de regressão. Isso demonstra a boa combinação da MVS com a Regressão.

A Rede Neural Artificial é uma técnica de AM amplamente utilizada na área. Uma de suas muitas aplicações é o planejamento de caminhos, pois ela tem uma grande capacidade de aprender caminhos não lineares (Ahmad et al., 2013). Dois dos trabalhos selecionados apresentam o uso de Rede Neural Artificial, o que corresponde à 16,6\% do total. Essa Rede Neural Artificial foi usada em conjunto com a tarefa da Rede Elman por Ahmad et al. (2013), e Processamento de Linguagem Natural por Zielke et al. (2018). 
As Redes Bayesianas ganharam importância no mundo científico graças à sua utilidade na modelagem e tratamento em incerteza, principalmente na Medicina (Kincaid, Hamilton, Tarr, \& Sangani, 2003). As Redes Bayesianas podem ser adequadas no uso de simuladores de casos clínicos. Pearl (2014) sugeriu que o raciocínio humano deve adotar uma estratégia diferente, que desvie o foco da faceta quantitativa da representação das probabilidades, para dar mais atenção às relações de dependência entre variáveis. Com base nisso, a estrutura de conhecimento utilizada para avaliação humana é do tipo de gráficos de dependência e percorrer as conexões entre seus nós consiste nos processos básicos de pesquisa, o que fazem as Redes Bayesianas (Flores et al., 2013). As redes Bayesianas são adequadas para modelar o conhecimento e apoiar o raciocínio sob a modelagem do conhecimento de incerteza (Flores et al., 2013).

Apenas um dos trabalhos encontrados apresentou de forma clara o uso de Redes Bayesianas, que corresponde a 8,3\% do total de trabalhos selecionados. O trabalho apresentado por Flores et al. (2013) utilizou as Redes Bayesianas, mas não especificou a tarefa de AM utilizado. O trabalho usou o aprendizado para modelar conhecimento e apoiar o raciocínio sob a modelagem do conhecimento de incerteza, o que se alinha a um dos objetivos das Redes Bayesianas. O trabalho obteve uma boa validação e seguiu o processo da ISO / IEC 14598-6.

Dentre as tarefas de AM, 41,7\% dos trabalhos analisados apresentam o uso de Regressão Linear. Rede Elman, Processamento de Linguagem Natural, Regressão Vetorial, Regressão Logística e Q-Learning são apresentados em 8,3\% dos trabalhos, e 16,7\% dos trabalhos não informaram a tarefa utilizada. Assim, pode-se observar que a MVS, Rede Neural Artificial e as Redes Bayesiana são as principais técnicas de AM nos Jogos para Medicina, pois encontram-se em 50\% dos trabalhos.

\subsection{Quais os principais jogos sérios que utilizam aprendizagem de máquina na área da medicina?}

Para identificação dos principais jogos que usaram aprendizagem de máquina na área da medicina foram levados em conta alguns critérios de qualidade como qualis e número de páginas, além do amadurecimento da pesquisa por meio de outras publicações, validação do jogo, limitações da pesquisa, vantagens e outros pontos importantes da pesquisa. Assim, chegou-se a conclusão de que os principais jogos que utilizam AM na área da medicina são o FOCUS e SimDeCS.

O FOCUS, apresentado por Alchalabi et al. (2017) (2018), teve o objetivo de treinar e fortalecer a capacidade de atenção dos pacientes com TDAH e detectar seu nível de atenção. Este trabalho teve duas publicações inclusas nessa RSL com qualis A1 e B3, e 9 e 6 páginas. Foi validado usando 9 usuários, 4 com TDAH e 5 Saudáveis, com uma precisão de até $96 \%$ e $98 \%$ na classificação dos dados do EEG para detectar o estado de atenção correto durante o jogo em indivíduos saudáveis e com TDAH, respectivamente. Assim, esse trabalho apresenta um novo método para classificação de dados de EEG e possibilita a identificação de pessoas com TDAH. É apresentado, segundo os autores, como o primeiro trabalho que estabeleceu as bases para a integração de um classificador de AM com um jogo sério para detectar o TDAH e como primeiro trabalho na literatura de instrumentação e medição que tentou medir a atenção para detectar o TDAH. Este Jogo é controlado por meio de EMOTIV. Os dados EEG brutos foram extraídos e registrados 
durante as sessões de teste usando scripts Python executados em segundo plano e os modelos de classificação foram construídos também usando Python.

O SimDeCS (Simulação para Tomada de Decisão no Serviço de Saúde), apresentado por Flores et al. (2013), teve o objetivo de realizar o monitoramento do estudante de medicina durante o processo de simulação, fornecendo feedback e orientações sobre as decisões clínicas tomadas. Esse trabalho tem qualis B3, e 6 páginas. Foi validado com 24 pessoas, sendo 13 médicos, 05 professores, 05 alunos de graduação e 01 aluno de pós-graduação, usando o software e preenchendo questionário. Também foi avaliado em termos de qualidade técnica e usabilidade, obedecendo à norma brasileira ISO / IEC 14598-6, que recomenda um mínimo de oito avaliadores (ABNT, 2004). O jogo trabalha o desenvolvimento das capacidades técnicas e competência no diagnóstico formulado, seguindo o próprio ritmo de aprendizagem do usuário/jogador. O processo de formulação do diagnóstico médico pode ser visto como um conjunto de etapas como: entrevista médica, exame físico, formulação de hipóteses diagnósticas e requisição (ou não) de exames complementares. Várias pessoas se dedicaram a esse propósito, contando com profissionais da área de saúde para modelar o conhecimento específico em Redes Bayesianas, o qual inclui também especialistas em modelagem de área computacional e especialistas na educação, todos trabalhando com táticas pedagógicas para avançar junto com profissionais no desenvolvimento. Quanto ao próprio sistema, está em fase final de desenvolvimento com três redes (dor de cabeça, dispepsia e parasitoses), possibilitando moldar cerca de 80 casos clínicos por professores que se preocupam em delinear cada caso pessoal. Apenas dez casos clínicos foram preparados para os estudantes se exercitarem.

\subsection{Quais as principais áreas da medicina em que os jogos estão sendo aplicados?}

Os trabalhos foram classificados quanto as temáticas da medicina mais abordadas, sendo divididos em: Casos clínicos; Atenção; Cirurgias; Interação Médico e Paciente; Gastos Energéticos Corporal; e, Cognição. Na Figura 3, é apresentada a distribuição dos trabalhos nas temáticas.

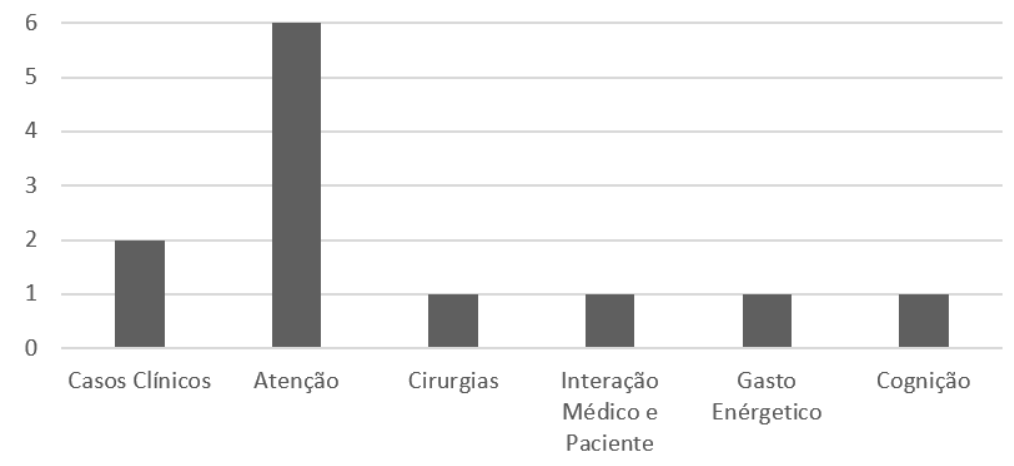

Figura 3. Áreas da saúde dos jogos

Seis trabalhos estão relacionados à Atenção. Chouhan et al. (2015) trabalharam a atenção e memória por meio de um jogo com intuito de memorizar uma sequência de objetos e, em seguida, seleciona-los corretamente na ordem de sua aparência para as diferentes modalidades de estímulos. O jogo apresentou melhoria nos níveis de atenção dos jogadores. Perdiz et al. (2018) focaram em aumentar o nível de atenção dos jogadores por meio do uso de movimentos oculares para controlar um Card, apesar de apresentar 
uma melhora relativa na atenção, não houve efeito perceptivos nos scores dos jogadores. George et al. (2011) tiveram o objetivo de melhorar a atenção e relaxamento. O trabalho foi validado com dez usuários e usou dados de EEG para medir a atenção e relaxamento do usuário, apresentando melhora de atenção e relaxamento dos usuários. Esse trabalho ainda apresentou configurações adequadas para interação com videogames simples e utilizou o software de aprendizado Matlab para uso da Regressão Linear.

Desses seis trabalhos, três estão ligados ao Transtorno do Déficit de Atenção com Hiperatividade (TDAH). O jogo Groundskeeper, apresentado por Heller et al. (2013), objetivou construir modelos para prever o funcionamento executivo de desordens, com dados coletados no jogo dos sujeitos de teste. Foram abordados TDAH, transtorno depressivo, transtornos de ansiedade, transtorno desafiador de oposição, transtorno do pânico ou transtornos alimentares. Esse jogo usa Sifteo Cubes e coleta dados de respostas dos usuários durante o jogo. Usou o AMS, através da Regressão Linear, e usou o software Weka para o aprendizado. O trabalho identificou déficits de desempenho executivo e diferenciação baseada em padrões de comportamento entre TDAH, tipo combinado, TDAH, tipo desatento, ansiedade e depressão. O jogo FOCUS também apresentou em seus dois trabalhos identificação de atenção e TDAH.

Dois dos trabalhos selecionados apresentaram casos clínicos. O trabalho apresentado por Lima et al. (2016) apresentou o DocTraining, um jogo para auxiliar alunos e professores de medicina como forma de mitigar o crescente número de erros médicos em casos clínicos. Esse trabalho usou aprendizado na identificação das doenças de casos clínicos e diabetes no jogo, e usou o software weka além de usar Sistema multiagente. Apesar da variedade de utilidades abordadas no trabalho, o jogo encontra-se em teste alfa. O trabalho apresentado por Flores et al. (2013) focou em Decisões Clínicas e trabalhou as capacidades técnicas do usuário.

Baseado nesses resultados, podemos identificar que as principais áreas da saúde com jogos que utilizam AM é a área de Atenção, por ser tratado em metade dos trabalhos encontrados com foco em Transtorno do Déficit de Atenção e Hiperatividade, e os Casos Clínicos. Além das áreas de Cirurgias, Interação Médico e Paciente, Gasto Energético Corporal e Cognição.

\section{Conclusões e Limitações}

Nesta pesquisa foi apresentado o planejamento, a condução e os resultados de uma RSL sobre o AM nos jogos para medicina, publicados nos últimos 10 anos, no âmbito internacional. Os trabalhos foram pesquisados nas bases de dados da IEEE, ACM Digital Library, Science Direct e Scopus. A busca pelos trabalhos resultou na pré-seleção de 40 trabalhos, dentre os quais 12 foram incluídos para a extração de dados.

Assim, foi possível observar que o AM nos jogos para medicina possui aplicação no AMS, através do uso de MVS, Rede Neural Artificial e Redes Bayesianas, e com foco na tarefa de Regressão Linear. Esses jogos focaram principalmente na área da Memória e Casos clínicos. Foi perceptível que mesmo com o avanço dessa tecnologia, ainda foi pouco explorada, pois com base na leitura dos trabalhos foi observado que ainda existem várias áreas da medicina que foram pouco exploradas e poucas técnicas de AM utilizadas. Não foi encontrada nenhum trabalho que utilizasse múltiplos classificados, o que poderia maximizar os resultados, por meio da combinação de classificadores. 
Por meio dos resultados encontrados, foi possível mapear as técnicas utilizadas, os principais jogos, o principal tipo de AM utilizado e as principais áreas abordadas. Porém, apenas uma pequena parcela desses trabalhos fizeram o uso do AM nos jogos para medicina, o que se tornou uma limitação deste trabalho. Em resumo, os resultados apresentados nesta RSL apresentam as seguintes contribuições: fornecem uma visão geral do AM; fornecem características (requisitos) usadas nos trabalhos selecionados; e, identificam técnicas que podem ser utilizadas em novos jogos.

\section{Agradecimentos}

Os agradecimentos são pelo fomento da Coordenação de Aperfeiçoamento de Pessoal de Nível Superior (CAPES) no Programa de Pós-Graduação em Ciência da Computação da Universidade Federal Rural do Semi-Árido e da Universidade do Estado do Rio Grande do Norte.

\section{Referências}

ABNT. (2004). Abnt nbr iso/iec 14598-6: Engenharia de software-avaliação de produtoparte 6: documentação de módulos de avaliação. Author.

Abt, C. C. (1987). Serious games. University press of America.

Ahmad, M. A., Mansoor, S. B., Khan, Z. A., Aqeel, W. A., Kabir, S. H. (2013). Benchmarking expert surgeons' path for evaluating a trainee surgeon's performance. In Proceedings of the 12th acm siggraph international conference on virtual-reality continuum and its applications in industry (pp. 57-62).

Alchalabi, A. E., Elsharnouby, M., Shirmohammadi, S., Eddin, A. N. (2017). Feasibility of detecting adhd patients' attention levels by classifying their eeg signals. In $\mathrm{Me}$ dical measurements and applications (memea), 2017 ieee international symposium on (pp. 314-319).

Alchalabi, A. E., Shirmohammadi, S., Eddin, A. N., Elsharnouby, M. (2018). Focus: Detecting adhd patients by an eeg-based serious game. Ieee Transactions on Instrumentation and Measurement.

Chouhan, T., Panse, A., Smitha, K. G., Vinod, A. P. (2015). A comparative study on the effect of audio and visual stimuli for enhancing attention and memory in brain computer interface system. In Systems, man, and cybernetics (smc), 2015 ieee international conference on (pp. 3104-3109).

Clua, E. W. G. (2014). Jogos sérios aplicados a saúde. Journal of Health Informatics, 6.

Deo, R. C. (2015). Machine learning in medicine. Circulation, 132(20), 1920-1930.

Deo, R. C., Musso, G., Tasan, M., Tang, P., Poon, A., Yuan, C., ... others (2014). Prioritizing causal disease genes using unbiased genomic features. Genome biology, $15(12), 534$.

Faceli, K., Lorena, A., Gama, J., Carvalho, A. (2011). Inteligência artificial-uma abordagem de aprendizado de máquina. Rio de Janeiro: LTC.

Flores, C. D., Barros, P., Cazella, S., Bez, M. R. (2013). Leveraging the learning process in health through clinical cases simulator. In Serious games and applications for health (segah), 2013 ieee 2nd international conference on (pp. 1-6).

Frutos-Pascual, M., Zapirain, B. G. (2017, June). Review of the use of ai techniques in serious games: Decision making and machine learning. Ieee Transactions on Computational Intelligence and AI in Games, 9(2), 133-152. doi: 10.1109/TCIAIG .2015 .2512592 
Galvão, T. F., Pereira, M. G. (2014). Revisões sistemáticas da literatura: passos para sua elaboração. Epidemiologia e Serviços de Saúde, 23, 183-184.

George, L., Lotte, F., Abad, R. V., Lécuyer, A. (2011). Using scalp electrical biosignals to control an object by concentration and relaxation tasks: design and evaluation. arXiv preprint arXiv:1111.5285.

Heller, M. D., Roots, K., Srivastava, S., Schumann, J., Srivastava, J., Hale, T. S. (2013). A machine learning-based analysis of game data for attention deficit hyperactivity disorder assessment. GAMES FOR HEALTH: Research, Development, and Clinical Applications, 2(5), 291-298.

Hervás, R., Johnson, E., de la Franca, C. G. L., Bravo, J., Mondéjar, T. (2016). A learning system to support social and empathy disorders diagnosis through affective avatars. In Ubiquitous computing and communications and 2016 international symposium on cyberspace and security (iucc-css), international conference on (pp. 93-100).

Kim, M., Angermann, J., Bebis, G., Folmer, E. (2013). Vizical: Accurate energy expenditure prediction for playing exergames. In Proceedings of the 26th annual acm symposium on user interface software and technology (pp. 397-404).

Kincaid, J. P., Hamilton, R., Tarr, R. W., Sangani, H. (2003). Simulation in education and training. In Applied system simulation (pp. 437-456). Springer.

Kitchenham, B. (2004). Procedures for performing systematic reviews (Keele University. Technical Report TR/SE-0401). Department of Computer Science, Keele University, UK.

Lima, R. M., Santos, A. M., Mendes Neto, F. M., Sousa Neto, A. F., Leão, F. C. P., Macedo, F. T., Canuto, A. M. P. (2016, May). A 3d serious game for medical students training in clinical cases. In 2016 ieee international conference on serious games and applications for health (segah) (p. 1-9). doi: 10.1109/SeGAH.2016 .7586255

Luger, G. F. (2014). Artificial intelligence. Pearson Education.

Matsubara, E. T. (2004). O algoritmo de aprendizado semi-supervisionado co-training e sua aplicação na rotulação de documentos (Unpublished doctoral dissertation). Universidade de São Paulo.

Monard, M. C., Baranauskas, J. A. (2003). Conceitos sobre aprendizado de máquina. Sistemas Inteligentes-Fundamentos e Aplicações, 1(1), 32.

Norvig, P., Russell, S. (2014). Inteligência artificial: Tradução da 3a edição (Vol. 1). Elsevier Brasil.

Pearl, J. (2014). Probabilistic reasoning in intelligent systems: networks of plausible inference. Elsevier.

Perdiz, J., Garrote, L., Pires, G., Nunes, U. J. (2018, May). Measuring the impact of reinforcement learning on an electrooculography-only computer game. In 2018 ieee 6th international conference on serious games and applications for health (segah) (p. 1-8). doi: 10.1109/SeGAH.2018.8401359

Zielke, M. A., Zakhidov, D., Hardee, G. M., Pradeep, J., Evans, L., Lodhi, Z., ... Ward, E. (2018). Exploring medical cyberlearning for work at the human/technology frontier with the mixed-reality emotive virtual human system platform. In 2018 ieee 6th international conference on serious games and applications for health (segah) (pp. $1-8)$. 